\title{
DOR EM UNIDADE NEONATAL: CONHECIMENTO, ATITUDE E PRÁTICA DA EQUIPE DE ENFERMAGEM*
}

\author{
Ana Carla de Oliveira Soares ${ }^{1}$, Maria de Fátima Costa Caminha², Ana Cláudia Ferreira Pinheiro Coutinho \\ Claudiane Maria Urbano Ventura ${ }^{4}$
}

\begin{abstract}
RESUMO: Avaliar conhecimento, atitude e prática da equipe de enfermagem no manejo da dor no recémnascido, segundo a formação profissional. Estudo transversal, realizado na Unidade Neonatal em hospital de referência em Recife, estado de Pernambuco, com 105 profissionais, entre agosto e outubro de 2014. Análise realizada no Stata 12.1, utilizado teste t-Student e considerado $p<0,05$. Quando comparado conhecimento, atitude e prática da equipe de enfermagem, houve melhor desempenho para atitude, embora não tenha sido estatisticamente significante. Em conhecimento e prática, no nível superior, o valor foi significativo para os que receberam treinamento sobre manejo da dor. No nível médio, todas as diferenças de médias foram significantes $(p<0,05)$. Para todos os profissionais, a atitude apresentou maiores médias. Conclui-se que os profissionais que trabalham com o recém-nascido devem ser capacitados e treinados continuamente, para que o conhecimento teórico reflita sobre a prática profissional.
\end{abstract}

DESCRITORES: Manejo da dor; Recém-nascido; Equipe de Enfermagem; Unidades de Terapia Intensiva Neonatal.

\section{PAIN IN THE NEONATAL UNIT: THE KNOWLEDGE, ATTITUDE AND PRACTICE OF THE NURSING TEAM}

\begin{abstract}
This study aims to evaluate the knowledge, attitude and practice of the nursing team in the management of pain in the newborn, in terms of professional training. It is a transversal study, undertaken in the Neonatal Unit of a specialist hospital in Recife in the Brazilian state of Pernambuco, with 105 professionals, between August and October 2014. Analysis was undertaken using the Stata 12.1 software, Student's t-test was used and $p<0.05$ was considered. When the knowledge, attitude and practice of the nursing team was compared, there was better performance for attitude, although this was not statistically significant. In knowledge and practice, among those with higher education, the value was significant for those who had received training on pain management. For those educated to high school level only, all of the differences of means were significant $(p<0.05)$. For all the professionals, attitude presented higher means. It is concluded that the professionals who work with the newborn must be continuously empowered and trained, such that theoretical knowledge may be reflected in the professional practice.
\end{abstract}

DESCRIPTORS: Pain Management; Infant, newborn; Nursing, team; Intensive care units, neonatal.

\section{DOLOR EN UNIDAD NEONATAL: CONOCIMIENTO, ACTITUD Y PRÁCTICA DEL EQUIPO DE ENFERMERÍA}

RESUMEN: Estudio cuya finalidad fue evaluar conocimiento, actitud y práctica del equipo de enfermería en el manejo del dolor del recién nacido, de acuerdo con la formación profesional. Estudio transversal, realizado en Unidad Neonatal en hospital de referencia en Recife, estado de Pernambuco, con 105 profesionales, entre agosto y octubre de 2014. Análisis realizado en el Stata 12.1, utilizando test $\mathrm{t}$-Student y considerando $\mathrm{p}<0,05$. Si comparados conocimiento, actitud y práctica del equipo de enfermería, hubo mejor desempeño para actitud, a pesar de no ser estadísticamente significante. Acerca de conocimiento y práctica, en nivel superior, el valor fue significativo para los que tuvieron entrenamiento del manejo del dor. En nivel medio, todas las diferencias de medias fueron significantes $(p<0,05)$. Para todos los profesionales, la actitude presentó medias mayores. Se concluye que los profesionales que trabajan con el recién nacido deben ser habilitados y entrenados continuamente, para que el conocimiento teórico sea reflejo sobre la práctica profesional.

DESCRIPTORES: Manejo del dolor; Recién nacido; Equipo de Enfermería; Unidades de Terapia Intensiva Neonatal.

\footnotetext{
*Artigo extraído da dissertação intitulada: "Manejo da dor no recém-nascido em Unidade Neonatal: conhecimento, atitude e prática da equipe de Enfermagem". Instituto de Medicina Integral Prof. Fernando Figueira, 2015.

${ }^{1}$ Enfermeira. Mestre em Cuidados Intensivos. Docente do Centro Universitário Tiradentes. Maceió, AL, Brasil.

Enfermeira. Doutora em Nutrição. Secretária Executiva do Conselho Científico do Instituto de Medicina Integral Prof. Fernando Figueira. Recife, PE, Brasil.

${ }^{3}$ Enfermeira. Mestre em Saúde Ambiente. Coordenadora do Programa de Residências em Enfermagem da Universidade Estadual de Ciências da Saúde de Alagoas. Maceió, AL, Brasil.

${ }^{4}$ Enfermeira. Mestre em Saúde Materno-Infantil. Enfermeira gerente da Unidade Neonatal do Instituto de Medicina Integral Prof. Fernando Figueira. Recife, PE, Brasil.
}

\author{
Autor Correspondente: \\ Ana Carla de Oliveira Soares \\ Centro Universitário Tiradentes \\ Cj José Tenório, bloco 55, ap 002 - 57046-350 - Maceió, AL, Brasil \\ E-mail: anacos87@gmail.com
}

Recebido: $17 / 11 / 2015$ 


\section{INTRODUÇÃO}

A dor não é apenas uma sensação desagradável, mas também modalidade sensorial essencial à sobrevivência, e quando não tratada causa sofrimento aos indivíduos afetados ${ }^{(1)}$. Durante muito tempo, profissionais da saúde acreditaram que o recém-nascido $(R N)$ não sentia dor, por ser protegido pela imaturidade do sistema nervoso central. No início da década de 60, foi observado que a transmissão dos impulsos pelo trato sensorial acontecia mesmo com a mielinização incompleta do sistema nervoso ${ }^{(2)}$.

É conhecido que nas Unidades de Terapia Intensivas Neonatais (UTIN) os RNs prematuros ou gravemente doentes são expostos a múltiplos procedimentos dolorosos como punção de calcâneo e venosa repetida e prolongada ${ }^{(3)}$. Sendo assim, são utilizadas escalas que tentam analisar alterações comportamentais e fisiológicas à sua dor ${ }^{(4)}$.

Após a avaliação da dor, a equipe de enfermagem deve atuar terapeuticamente utilizando medidas não farmacológicas ${ }^{(5)}$, como redução da luminosidade, diminuição de ruídos, enrolamento, contenção, sucção não-nutritiva, glicose e agrupamento dos cuidados $^{(3)}$.

Muitos fatores influenciam a prática para o manejo da dor no RN, como falta de conhecimento, atitude, sobrecarga de trabalho, experiência profissional e pessoal. O sucesso da transferência do conhecimento na prática depende da qualidade da evidência, contexto receptivo a mudanças ${ }^{(6)}$.

A dor é considerada sinal vital e muitas vezes é subestimada, não avaliada e não tratada, principalmente em RNs internados em Unidade Neonatal (UN), que tem maneiras específicas de expressá-la. A falta de conhecimento e a atitude dos profissionais de enfermagem podem ser os principais motivos para o não tratamento da dor. Então, para uma possível transformação da assistência, foi importante realizar este estudo, e assim obter informações relevantes quanto ao manejo da dor no RN em UN.

Desta forma, o estudo teve como objetivo avaliar conhecimento, atitude e prática da equipe de enfermagem, quanto ao manejo da dor no RN em UN, segundo a formação dos profissionais.

\section{MÉTODO}

Estudo avaliativo, tipo inquérito Conhecimento, Atitude e Prática (CAP), corte transversal e abordagem quantitativa, realizado na UN de um hospital de referência em saúde materno-infantil, em Recife, estado de Pernambuco.

A amostra do estudo foi não-probabilística, por conveniência, composta por 105 profissionais de enfermagem (15 enfermeiras e 90 técnicos de enfermagem). A população foi de 132 profissionais (115 técnicos de enfermagem e 17 enfermeiras), sendo excluídos 15 profissionais; houve sete recusas e cinco perdas. O critério de inclusão foi: enfermeiras e técnicos de enfermagem lotados na UN no período da coleta de dados e que aceitaram participar da pesquisa. Os critérios de exclusão foram: enfermeiras e técnicos de enfermagem em cumprimento de licença médica, férias ou afastamento do serviço; aqueles que cobrem férias e prestadores de serviço; enfermeira autora da pesquisa.

A coleta de dados foi realizada no período de agosto a outubro de 2014, por meio do contato pessoal da pesquisadora com a equipe de enfermagem no início de cada plantão, com questionários distribuídos aos profissionais que concordaram em participar do estudo, mediante a assinatura do Termo de Consentimento Livre e Esclarecido. O preenchimento foi realizado no local do trabalho, não sendo permitida consulta a fontes de informação, aguardando o preenchimento pelo participante da pesquisa.

O questionário abordava perguntas que contemplavam características profissionais e o inquérito CAP, que avaliou três esferas: Conhecimento (capacidade de adquirir e reter informações a serem utilizadas, uma mistura de compreensão, experiência, discernimento e habilidade), Atitude (inclinação para reagir de certo modo a certas situações; ver e interpretar eventos de acordo com certas predisposições; organizar opiniões dentro de uma estrutura inter-relacionada e coerente) e Prática (aplicação de regras e conhecimentos que levam à execução da ação e maneira ética). Este inquérito foi constituído de três etapas de acordo com a metodologia de estudo semelhante ${ }^{(7)}$ : 
Primeira etapa: construção do questionário inicial. Foi elaborado baseado em estudos que analisaram conhecimentos da equipe de enfermagem em relação à avaliação e terapias não-farmacológicas e farmacológicas para alívio da dor no RN em UTIN ${ }^{(6,8-9)}$.

Segunda etapa: avaliação da aparência e conteúdo do questionário realizado por três enfermeiras, com mais de 10 anos de atuação na área de Cuidados Intensivos de três instituições de Ensino Superior em Enfermagem da cidade de Recife. As perguntas foram pontuadas de acordo com o grau de importância para construção do questionário ( $0=$ Desnecessário, $1=$ Regular, 2=Bom, 3=Ótimo), de forma a permanecerem aquelas que obtiveram pontuação de no mínimo dois, após duas avaliações do instrumento.

Terceira etapa: testagem prévia do instrumento, com 20 profissionais de enfermagem que não participaram do estudo, para avaliação quanto à objetividade, clareza das questões e tempo de preenchimento do questionário. As perguntas do questionário valeram de 0 a 3 ( $0=$ Desnecessário, 1=Regular, 2=Bom, 3=Ótimo), permanecendo aquelas que obtiveram pontuação de no mínimo dois em todas as avaliações.

Para as questões sobre Conhecimento e Prática, foram atribuídas respostas dicotômicas ("sim" /" não"). Para atitude, utilizou-se a escala Likert de cinco níveis ("concordo"/"concordo plename nte"|"discordo"|"discordo plenamente"|"não tenho opinião").

Para análise dos dados, foi considerada para o Conhecimento: questão adequada quando a resposta foi "sim" para as assertivas verdadeiras, ou "não" para as falsas; questão inadequada quando a resposta foi "não" para assertivas verdadeiras, ou "sim" para falsas.

Em relação à Atitude: questão adequada quando a resposta foi "concordo"/"concordo plenamente" para assertivas verdadeiras ou "discordo"I"discordo plenamente" para falsas; questão não adequada quando a resposta foi "discordo"/"discordo plenamente"/"não tenho opinião" para assertivas verdadeiras ou "concordo"|"concordo plenamente"I"não tenho opinião" para falsas.

Quanto à Prática: questão adequada quando a resposta foi "sim" e questão inadequada quando a resposta foi "não". As respostas foram consideradas adequadas ou não adequadas de acordo com a literatura sobre manejo da dor em RN internados em UN.

Para cada seção foi calculada uma nota em escala de 0 a 10, sendo considerada nota máxima 10, quando todas as questões apresentarem respostas corretas. Para o Conhecimento, foi atribuído o valor de 0,9 para cada questão; Atitude, cada afirmativa obteve o valor de 1,6; e Prática, foi atribuído o valor de 2,0 para cada questão. Então, quanto maior o escore, maior o conhecimento, a atitude e a prática dos profissionais de enfermagem.

A digitação foi em dupla entrada no programa Excel, validado no Epilnfo 3.4 .3 e os dados analisados no Stata 12.1. Foi realizado o Teste t de Student para comparação das médias de acerto, considerando nível de significância quando $\mathrm{p}<0,05$. Os dados foram analisados e apresentados, considerando dois grupos de profissionais: nível superior (enfermeiro) e nível médio (técnico de enfermagem).

O projeto foi aprovado pelo Comitê de Ética em Pesquisa do IMIP, protocolo número 4154-14, conforme a Resolução $n^{\circ} 466 / 12^{(10)}$ do Ministério da Saúde.

\section{RESULTADOS}

Participaram do estudo 105 profissionais de enfermagem, sendo 14,3\% (15) enfermeiras e 85,7\% (90) técnicos de enfermagem. As enfermeiras tinham média de idade 34,1 anos (DP=7,3), sete (50\%) com mais de cinco anos de conclusão da graduação e oito $(53,4 \%)$ tinham mais de cinco anos de atuação em UN. Cerca de nove (60\%) mencionaram pós-graduação em Saúde da Criança/Neonatalogia e seis (40\%) referiram capacitação sobre manejo da dor no RN.

Observou-se que 98,1\% (89) dos técnicos de enfermagem era do sexo feminino, com média de idade 35,3 anos (DP=9,8), 49,4\% (37) com tempo de formação profissional acima de cinco anos e 38,4\% (33) tinham mais de cinco anos de atuação em UN. Cerca de $60 \%$ (51) referiram curso de especialização técnica e 65,6\% (59) mencionaram capacitação sobre manejo da dor no RN. 
Em relação às medidas não-farmacológicas para o manejo da dor, as mais citadas entre as enfermeiras foram glicose $25 \%$ e sucção não-nutritiva; entre os técnicos de enfermagem glicose $25 \%$ e contenção do RN com lençol.

Na Tabela 4, não foi observada diferença estatística entre as médias de conhecimento, atitude e prática e as características da formação de profissionais de enfermagem.

Na Tabela 5, verifica-se que, entre os profissionais de nível superior, a diferença entre as médias foi estatisticamente significante apenas para aqueles que receberam treinamento sobre manejo da dor. Em relação aos profissionais de nível médio, para todas as variáveis, as diferenças de médias se mostraram estatisticamente significantes.

Tabela 1 - Distribuição de respostas adequadas sobre o conhecimento em manejo da dor no RN da equipe de Enfermagem da UN em um Hospital de Referência. Recife, PE, Brasil, 2014

\begin{tabular}{lcccc}
\multirow{2}{*}{\multicolumn{1}{c}{ Aspectos }} & \multicolumn{2}{c}{ Nível Superior } & \multicolumn{2}{c}{ Nível Médio } \\
\cline { 2 - 5 } & Respostas adequadas & Respostas adequadas \\
\cline { 2 - 5 } & $\mathbf{n = 1 5}$ & $\mathbf{\%}$ & $\mathbf{n = 9 0}$ & $\mathbf{9}$ \\
\hline RN sente dor & 15 & 100 & 90 & 100 \\
\hline Maior tempo de UTIN torna o RN mais tolerante à dor & 8 & 53,3 & 61 & 67,8 \\
\hline Ausência de choro indica que o RN não está sentindo dor & 13 & 86,7 & 83 & 92,2 \\
\hline É importante tratar a dor no RN & 14 & 93,3 & 86 & 95,5 \\
\hline Tratar a dor melhora o prognóstico & 13 & 86,7 & 63 & 70 \\
\hline Tratar a dor diminui o sofrimento & 14 & 93,3 & 80 & 88,9 \\
\hline Tratar a dor diminui o stress & 14 & 93,3 & 75 & 83,3 \\
\hline Conhece alguma medida não-farmacológica & 13 & 86,7 & 50 & 55,6 \\
\hline Conhece alguma medida farmacológica & 12 & 80 & 55 & 61,1 \\
\hline A medida de alívio da dor deve ser registrada no prontuário & 15 & 100 & 88 & 97,8
\end{tabular}

Tabela 2 - Distribuição de respostas adequadas sobre a atitude em relação manejo da dor no RN da equipe de enfermagem da UN em um Hospital de Referência. Recife, PE, Brasil, 2014

\begin{tabular}{llccc} 
Aspectos & \multicolumn{2}{c}{ Nível Superior } & \multicolumn{2}{c}{ Nível Médio } \\
\cline { 2 - 5 } & Respostas adequadas & Respostas adequadas \\
\cline { 2 - 5 } & $\mathbf{n = 1 5}$ & $\mathbf{\%}$ & $\mathbf{n = 9 0}$ & $\mathbf{\%}$ \\
\hline O manejo da dor deve ser realizado apenas pela Enfermeira & 15 & 100 & 87 & 96,7 \\
\hline $\begin{array}{l}\text { O manejo da dor pode ser realizado por qualquer } \\
\text { profissional de Enfermagem }\end{array}$ & 15 & 100 & 85 & 94,4 \\
\hline Todo RN deve receber medidas para alívio da dor & 14 & 93,3 & 79 & 87,8 \\
\hline $\begin{array}{l}\text { Ao realizar procedimentos dolorosos devo promover o alívio } \\
\text { da dor }\end{array}$ & 15 & 100 & 88 & 97,8 \\
\hline $\begin{array}{l}\text { O manejo da dor deve ser realizado antes do procedimento } \\
\text { doloroso }\end{array}$ & 15 & 100 & 81 & 90 \\
\hline $\begin{array}{l}\text { Devo saber se há na prescrição médica, medicação para } \\
\text { alívio da dor }\end{array}$ & 13 & 86,7 & 89 & 98,9 \\
\end{tabular}


Tabela 3 - Distribuição de respostas adequadas sobre a prática no manejo da dor no RN da equipe de Enfermagem da UN em um Hospital de Referência. Recife, PE, Brasil, 2014

\begin{tabular}{lcccc}
\multicolumn{1}{c}{ Aspectos } & \multicolumn{2}{c}{ Nível Superior } & \multicolumn{2}{c}{ Nível Médio } \\
\cline { 2 - 5 } & Respostas adequadas & Respostas adequadas \\
\cline { 2 - 5 } & $\mathbf{n = 1 5}$ & $\mathbf{\%}$ & $\mathbf{n = 9 0}$ & $\mathbf{\%}$ \\
\hline Avalia a dor no RN de acordo com a escala protocolo da UM & 11 & 73,3 & 73 & 81,1 \\
\hline Realizou medidas para alívio da dor no RN & 15 & 100 & 87 & 96,7 \\
\hline $\begin{array}{l}\text { Antes de realizar procedimento doloroso promove o alívio } \\
\text { da dor }\end{array}$ & 15 & 100 & 87 & 96,7 \\
\hline $\begin{array}{l}\text { Registra no prontuário ou na evolução de Enfermagem a } \\
\text { medida utilizada }\end{array}$ & 6 & 40 & 74 & 82,2 \\
\hline $\begin{array}{l}\text { Utiliza as medidas farmacológicas somente sob prescrição } \\
\text { médica }\end{array}$ & 15 & 100 & 88 & 97,8
\end{tabular}

Tabela 4 - Distribuição das médias de conhecimento, atitude e prática sobre manejo da dor no RN segundo as características da formação de profissionais de enfermagem da UN em um Hospital de Referência. Recife, PE, Brasil, 2014

\begin{tabular}{|c|c|c|c|c|c|c|c|c|c|}
\hline \multirow[b]{2}{*}{ Características } & \multicolumn{3}{|c|}{ Conhecimento } & \multicolumn{3}{|c|}{ Atitude } & \multicolumn{3}{|c|}{ Prática } \\
\hline & Média & (DP) & p* $^{*}$ & Média & (DP) & $\mathbf{p}^{*}$ & Média & (DP) & $\mathbf{p}^{*}$ \\
\hline \multicolumn{10}{|c|}{ Nível Superior } \\
\hline \multicolumn{10}{|c|}{ Tempo de graduação } \\
\hline$\leq 5$ anos & 7,9 & $-0,9$ & 0,405 & 10 & 0 & 0,972 & 7,7 & $(0.8)$ & 0,115 \\
\hline$>5$ anos & 8,1 & $-0,3$ & & 9,3 & $-0,3$ & & 8,8 & $-0,4$ & \\
\hline \multicolumn{10}{|l|}{ Pós-graduação } \\
\hline Sim & 7,7 & $-0,7$ & 0,275 & 9,8 & $-0,2$ & 0,836 & 8 & $-0,6$ & 0,234 \\
\hline Não & 8,2 & $-0,3$ & & 9,4 & $-0,3$ & & 8,6 & $-0,4$ & \\
\hline \multicolumn{10}{|c|}{ Treinamento sobre manejo da dor } \\
\hline Sim & 7,6 & $-0,2$ & 0,283 & 9,4 & $-0,3$ & 0,163 & 9 & $-0,4$ & 0,913 \\
\hline Não & 8,1 & $-0,7$ & & 9,8 & $-0,2$ & & 7,7 & $-0,6$ & \\
\hline \multicolumn{10}{|c|}{ Tempo de atuação na UTIN } \\
\hline$\leq 5$ anos & 7,9 & $-0,9$ & 0,449 & 10 & 0 & 0,96 & 7,7 & $-0,8$ & 0,121 \\
\hline$>5$ anos & 8 & $-0,3$ & & 9,3 & $-0,3$ & & 8,8 & $-0,3$ & \\
\hline \multicolumn{10}{|c|}{ Nível Médio } \\
\hline \multicolumn{10}{|c|}{ Tempo de formação } \\
\hline$\leq 5$ anos & 7,5 & $-0,2$ & 0,801 & 9,5 & $-0,2$ & 0,735 & 9 & $-0,3$ & 0,36 \\
\hline$>5$ anos & 7,3 & $-0,2$ & & 9,4 & $-0,1$ & & 9,1 & $-0,2$ & \\
\hline \multicolumn{10}{|c|}{ Especialização técnica } \\
\hline Sim & 7,1 & $-0,2$ & 0,057 & 9,4 & $-0,1$ & 0,577 & 9,2 & $-0,2$ & 0,677 \\
\hline Não & 7,6 & $-0,2$ & & 9,4 & $-0,2$ & & 9 & $-0,3$ & \\
\hline \multicolumn{10}{|c|}{ Treinamento sobre manejo da dor } \\
\hline Sim & 7,6 & $-0,1$ & 0,981 & 9,3 & $-0,1$ & 0,098 & 9,2 & $-0,2$ & 0,888 \\
\hline Não & 6,9 & $-0,3$ & & 9,6 & $-0,2$ & & 8,8 & $-0,3$ & \\
\hline \multicolumn{10}{|c|}{ Tempo de atuação na UTIN } \\
\hline$\leq 5$ anos & 7,6 & $-0,2$ & 0,941 & 9,4 & $-0,1$ & 0,452 & 9 & $-0,2$ & 0,2 \\
\hline$>5$ anos & 7,1 & $-0,3$ & & 9,5 & $-0,2$ & & 9,3 & $-0,2$ & \\
\hline
\end{tabular}

*Teste t de Student 
Tabela 5 - Comparação das médias de conhecimento e prática sobre manejo da dor no RN segundo características de formação de profissionais de enfermagem da UN em um Hospital de Referência. Recife, PE, Brasil, 2014

\begin{tabular}{|c|c|c|c|c|c|c|c|}
\hline \multirow[b]{2}{*}{ Características } & \multicolumn{2}{|c|}{ Conhecimento } & \multicolumn{2}{|c|}{ Prática } & \multicolumn{2}{|c|}{ Diferença } & \multirow{2}{*}{$\begin{array}{c}\begin{array}{c}\text { Valor } \\
\text { de p }\end{array} \\
\left.{ }^{*}\right)\end{array}$} \\
\hline & Média & (DP) & Média & (DP) & Média & (DP) & \\
\hline \multicolumn{8}{|c|}{ Nível Superior } \\
\hline \multicolumn{8}{|c|}{ Tempo de graduação } \\
\hline$\leq 5$ anos & 7,9 & $-0,9$ & 7,7 & $(0.8)$ & 0,2 & $-1,4$ & 0,548 \\
\hline$>5$ anos & 8,1 & $-0,3$ & 8,8 & $-0,4$ & 0,7 & $-0,6$ & 0,154 \\
\hline \multicolumn{8}{|l|}{ Pós-graduação } \\
\hline Sim & 7,7 & $-0,7$ & 8 & $-0,6$ & 0,3 & $-1,2$ & 0,416 \\
\hline Não & 8,2 & $-0,3$ & 8,6 & $-0,4$ & 0,4 & $-0,6$ & 0,292 \\
\hline \multicolumn{8}{|c|}{ Treinamento sobre manejo da dor } \\
\hline Sim & 7,6 & $-0,2$ & 9 & $-0,4$ & 1,4 & $-0,5$ & 0,028 \\
\hline Não & 8,1 & $-0,7$ & 7,7 & $-0,6$ & 0,4 & $-1,1$ & 0,63 \\
\hline \multicolumn{8}{|c|}{ Tempo de atuação na UTIN } \\
\hline$\leq 5$ anos & 7,9 & $-0,9$ & 7,7 & $-0,8$ & 0,2 & $-1,4$ & 0,548 \\
\hline$>5$ anos & 8 & $-0,3$ & 8,8 & $-0,3$ & 0,8 & $-0,5$ & 0,117 \\
\hline \multicolumn{8}{|c|}{ Nível Médio } \\
\hline \multicolumn{8}{|c|}{ Tempo de formação } \\
\hline$\leq 5$ anos & 7,5 & $-0,2$ & 9 & $-0,3$ & 1,5 & $-0,3$ & 0 \\
\hline$>5$ anos & 7,3 & $-0,2$ & 9,1 & $-0,2$ & 1,8 & $-0,3$ & 0 \\
\hline \multicolumn{8}{|c|}{ Especialização técnica } \\
\hline Sim & 7,1 & $-0,2$ & 9,2 & $-0,2$ & 2,1 & $-0,2$ & 0 \\
\hline Não & 7,6 & $-0,2$ & 9 & $-0,3$ & 1,4 & $-0,3$ & 0 \\
\hline \multicolumn{8}{|c|}{ Treinamento sobre manejo da dor } \\
\hline Sim & 7,6 & $-0,1$ & 9,2 & $-0,2$ & 1,6 & $-0,2$ & 0 \\
\hline Não & 6,9 & $-0,3$ & 8,8 & $-0,3$ & 1,9 & $-0,4$ & 0 \\
\hline \multicolumn{8}{|c|}{ Tempo de atuação na UTIN } \\
\hline$\leq 5$ anos & 7,6 & $-0,2$ & 9 & $-0,2$ & 1,4 & $-0,2$ & 0 \\
\hline$>5$ anos & 7,1 & $-0,3$ & 9,3 & $-0,2$ & 2,1 & $-0,4$ & 0 \\
\hline
\end{tabular}

*Teste t de Student

\section{- DISCUSSÃO}

De acordo com os resultados desse estudo, observou-se predominância do sexo feminino entre as categorias, relacionado à questão desse gênero na enfermagem, reproduzindo sua característica histórica, exercida quase exclusivamente por mulheres ${ }^{(11)}$.

A pós-graduação em Saúde da Criança/Neonatologia foi prevalente entre enfermeiras, e a maioria dos técnicos de enfermagem possuía curso de especialização, o que demonstra a preocupação do profissional com sua qualificação. A formação do enfermeiro na neonatologia se configura como especialização de reconhecido valor pela complexidade da assistência prestada, pois o enfermeiro que coordena as ações de enfermagem deve estar preparado, já que compete a ele a liderança da equipe e a responsabilidade pela assistência de enfermagem ${ }^{(12)}$.

Por outro lado, não foi encontrada frequência adequada quanto a treinamento específico sobre manejo da dor no RN para os profissionais, apesar de mais prevalente entre os técnicos de enfermagem. A educação permanente em saúde é reconhecida como estratégia relevante, contribuindo para 
qualificação contínua dos profissionais, para que possam agir precocemente, adiantando-se às possíveis intercorrências e prevenindo complicações ${ }^{(13)}$. Pode-se supor que a instituição estudada não dê subsídios suficientes para realizar tal estratégia.

Nas questões relacionadas ao conhecimento, destaca-se $100 \%$ de acerto quando os profissionais foram interrogados se o RN sentia dor; resultado semelhante também foi encontrado em Alfenas, estado de Minas Gerais ${ }^{(14)}$, o que pode ser justificado pela facilidade de acesso às informações, possibilitando melhor conhecimento a respeito do tema.

Mais que metade das enfermeiras (53,3\%) e técnicos de enfermagem $(67,8 \%)$ acredita que o maior tempo de UTIN e a maior exposição a procedimentos dolorosos não tornam o RN mais tolerante à dor. A exposição repetida do RN prematuro a múltiplos procedimentos dolorosos culmina em estresse, maior gasto metabólico e esgotamento das reservas energéticas, desfavorecendo o ganho de peso e retardando sua recuperação(15). Esses profissionais podem ter adquirido esse conhecimento a partir de sua prática e experiência profissional no cuidado ao RN exposto à dor.

Apesar de o choro ser uma das principais formas de expressão que o RN está sentindo dor, sua ausência não indica que a dor não está presente. Nesse sentido, a maioria dos profissionais responderam adequadamente a esse questionamento, enfermeiras $(86,7 \%)$ e técnicos de enfermagem $(92,2 \%)$. Além do choro, outros parâmetros também devem ser avaliados: movimentos dos membros, olhos espremidos, fronte saliente, alteração na frequência cardíaca ${ }^{(16-17)}$, entre outras alterações fisiológicas e comportamentais.

Mais de $90 \%$ dos profissionais de enfermagem acreditam que é importante tratar a dor no RN; resultado semelhante foi encontrado em estudo realizado com 57 profissionais de saúde num hospital do interior paulista, no qual $98,2 \%$ dos profissionais consideraram importante tratar a dor do RN, atitude justificada pela melhora do prognóstico e redução do sofrimento e estresse ${ }^{(6)}$. Esse conhecimento é refletido na prática dos profissionais, pois $100 \%$ das enfermeiras e $96,7 \%$ dos técnicos de enfermagem afirmaram que promovem o alívio da dor antes de realizar procedimento doloroso.

As medidas não-farmacológicas mais citadas, dentre as enfermeiras $(86,7 \%)$ e técnicos de enfermagem $(55,6 \%)$ que referiram conhecer algum tipo dessas medidas, foram a glicose $25 \%$, sucção não-nutritiva e contenção do RN com lençol. Em estudo realizado com 25 profissionais de enfermagem numa UTIN de referência em Fortaleza, estado do Ceará, sucção não-nutritiva, glicose $25 \%$ e agasalhar o RN, foram as condutas mais citadas para prevenir a dor no $\mathrm{RN}^{(18)}$.

Apesar das enfermeiras $(100 \%)$ e técnicos de enfermagem $(97,8 \%)$, terem conhecimento que a medida de alívio da dor deve ser registrada no prontuário, este dado vai de encontro à seção prática, pois apenas $40 \%$ das enfermeiras e $82,2 \%$ dos técnicos fazem o registro no prontuário. No estudo realizado na UTIN de um Hospital Universitário de Cuiabá-MT, com 127 prontuários de RN, verificou-se que o uso de medidas não-farmacológicas não foi registrado em nenhum prontuário ${ }^{(19)}$. O profissional deve registrar os cuidados prestados em prontuários, que são fontes de informações e garantem a continuidade do cuidado.

Na seção atitude, as categorias profissionais apresentaram mais de $85 \%$ de acertos em todas as afirmativas. Vale ressaltar que $100 \%$ das enfermeiras e $97,8 \%$ dos técnicos de enfermagem responderam adequadamente à afirmativa "ao realizar procedimentos dolorosos devo promover o alívio da dor"; dado semelhante foi encontrado na seção prática, no qual $100 \%$ das enfermeiras e $96,7 \%$ dos técnicos afirmaram que promovem o alívio da dor antes de realizar procedimento doloroso.

Observa-se que existe preocupação dos profissionais com a assistência prestada ao RN diante da dor. Fato encontrado também num estudo qualitativo realizado com 10 enfermeiros numa UTIN de um hospital público de Fortaleza, evidenciando que as intervenções realizadas pelos enfermeiros para amenizar a dor no RN foram predominantemente as não-farmacológicas desenvolvidas antes e depois do procedimento doloroso ${ }^{(20)}$.

Ao considerar o conhecimento, a atitude e a prática à luz de algumas características de formação dos profissionais, como tempo de graduação e atuação em UTIN, realização de curso de pósgraduação/especialização técnica, e de treinamento específico em manejo da dor no RN, verificou-se melhor desempenho relacionado à Atitude, seguido da Prática e do Conhecimento para as categorias 
profissionais.

Em outro estudo realizado com a metodologia CAP sobre fototerapia, em duas maternidades de Recife, considerando também a característica de formação dos profissionais de enfermagem, observou melhor desempenho relacionado à prática, seguido de atitude e conhecimento para os grupos de profissionais ${ }^{(7)}$. Ressalta-se a importância de capacitações com ênfase teórica para equipe de enfermagem, pois em ambos os estudos, os profissionais obtiveram menores médias em relação ao conhecimento.

Em relação ao conhecimento e prática, os profissionais de nível superior com tempo de graduação e de atuação em UTIN acima de cinco anos, obtiveram maiores notas. É possível que um maior tempo de formação e, consequentemente, uma maior experiência prática desses profissionais, tenha influenciado para melhor desempenho. Resultado diferente encontrado entre os profissionais de nível médio, pois os que obtiveram melhores médias foram os que tinham cinco anos ou menos de tempo de formado e atuação em UTIN. Neste caso, pode estar relacionado ao fato de que o conhecimento e prática aprendidos durante sua formação estão mais atualizados para aqueles que concluíram o curso recentemente.

É possível dizer que o tempo de formação profissional é importante para análise do perfil dos profissionais que trabalham em Unidade de Terapia Intensiva (UTI). O enfermeiro pode ter longa experiência em outra área, mas não dominar os elementos necessários ao cuidado do cliente sob cuidados intensivos, podendo, pela falta de experiência nessa área, ser considerado novato na UTI ${ }^{(21)}$.

A comparação entre as médias de conhecimento e prática das enfermeiras foi significativa estatisticamente apenas para aquelas que receberam treinamento sobre manejo da dor. Porém, para técnicos de enfermagem, todas as comparações de médias obtiveram significância estatística. Esses achados podem estar relacionados ao fato de que muitas vezes as capacitações oferecidas aos profissionais abordam mais as questões práticas que o conhecimento teórico, podendo aproximar à prática e distanciar do conhecimento desses profissionais.

Estudo realizado com 57 profissionais de saúde, num hospital do interior paulista, evidenciou que os profissionais reconhecem que os RNs sentem dor e que é necessário tratá-la. Porém, ainda existe uma lacuna para aplicação adequada de conhecimentos, pois perceberam o desconhecimento dos profissionais sobre avaliação e tratamento da dor decorrente de procedimentos dolorosos ${ }^{(6)}$.

\section{CONCLUSÃO}

A maioria das enfermeiras e técnicos de enfermagem apresentaram respostas mais satisfatórias quanto à atitude e à prática, consequentemente melhores médias. O conhecimento científico e a prática devem estar sempre relacionados, porém nem sempre isso acontece, como pode ser observado no estudo, quando enfermeiras que receberam treinamento sobre manejo da dor no RN obtiveram melhor média na prática, quando comparada ao conhecimento.

O estudo apresentou algumas limitações que devem ser consideradas. Apesar dos critérios adotados para construção do instrumento de coleta de dados, é possível que o formato das questões tenha induzido algumas respostas. Mesmo os profissionais estando cientes do objetivo do estudo, é possível que tenham sido tendenciosos ao afirmarem que realizam determinadas práticas. A avaliação da atitude e da prática baseada nas informações dos profissionais, sem a observação direta dos mesmos no manejo da dor no RN, é uma limitação que também deve ser considerada.

Apesar dessas limitações, o estudo apresentou resultados que podem ser úteis para organização da UN, no que se refere ao manejo da dor no RN, que podem levar à realização de novas pesquisas com outras metodologias. As lacunas de conhecimento, atitude e prática observadas podem contribuir na identificação de pontos a serem desenvolvidos e enfatizados na educação continuada para profissionais de enfermagem.

O manejo da dor no RN deve ser sempre considerado, principalmente quando se fala em UTIN. Os profissionais que trabalham com esse paciente devem ser capacitados e treinados continuamente, de forma que o conhecimento teórico reflita sobre a prática profissional, proporcionando assistência adequada, qualificada e humanizada ao RN. 
1. Kopf A, Patel NB. Guia para o Tratamento da Dor em Contextos de Poucos Recursos. Associação internacional para o estudo da dor (IASP) ${ }^{\circledR}$. [Internet] 2010 [acesso em 07 dez 2014]. Disponível: http://www.iasp-pain.org/files/ Content/ContentFolders/Publications2/FreeBooks/GuidetoPainManagement_Portuguese.pdf.

2. Brummelte S, Grunau RE, Chau V, Poskitt KJ, Brant R, Vinall J, et al. Procedural pain and brain development in premature newborns. Ann Neurol. [Internet] 2012; 71(3) [acesso em 07 dez 2014]. Disponível: http://dx.doi. org/10.1002/ana.22267.

3. de Aquino FM, Christoffel MM. Dor neonatal: medidas não-farmacológicas utilizada pela equipe de Enfermagem. Rev. Rene. [Internet] 2010; 11(n.esp) [acesso em 08 dez 2014]. Disponível: http://www.revistarene. ufc.br/edicaoespecial/a19v11esp_n4.pdf.

4. Guinsburg R, Cuenca MC. A linguagem da dor no recém-nascido. Documento Científico do Departamento de Neonatologia. Sociedade Brasileira de Pediatria. [Internet] 2010 [acesso em 08 dez 2014]. Disponível: http://www. sbp.com.br/pdfs/doc_linguagem-da-dor-out2010.pdf.

5. dos Santos LM, Ribeiro IS, Santana RCB. Identificação e tratamento da dor no recém-nascido prematuro na Unidade de terapia Intensiva. Rev. bras. enferm. [Internet] 2012; 65(2) [acesso em 08 dez 2014]. Disponível: http:// dx.doi.org/10.1590/S0034-71672012000200011.

6. Capellini VK, Daré MF, Castral TC, Christoffel MM, Leite AM, Scochi CGS. Conhecimento e atitudes de profissionais de saúde sobre avaliação e manejo da dor neonatal. Rev. Eletr. Enf. [Internet] 2014; 16(2) [acesso em 27 fev 2015]. Disponível: http://dx.doi.org/10.5216/ree.v16i2.23611.

7. Machado SPC, Samico IC, Braga TDA. Conhecimento, atitude e prática sobre fototerapia entre profissionais de Enfermagem de hospitais de ensino. Rev. bras. enferm. [Internet] 2012; 65(1) [acesso em 10 dez 2014]. Disponível: http://dx.doi.org/10.1590/S0034-71672012000100005.

8. Crescêncio EP, Zanelato S, Leventhal LC. Avaliação e alívio da dor no recém-nascido. Rev. Eletr. Enf. [Internet] 2009; 11(1) [acesso em 15 mai 2014]. Disponível: http://www.fen.ufg.br/fen_revista/v11/n1/pdf/v11n1a08.pdf.

9. Martins SW, Dias FS, Enumo SRF, de Paula KMP. Pain assessment and control by nurses of a neonatal intensive care unit. Rev. dor. [Internet] 2013; 14(1) [acesso em 15 mai 2014]. Disponível: http://dx.doi.org/10.1590/S180600132013000100006.

10. Ministério da Saúde (BR). Conselho Nacional de Saúde. Diretrizes e normas regulamentadoras de pesquisa envolvendo seres humanos. Resolução n. 466, de 12 de dezembro de 2012. Brasília; 2012.

11. Silva LG, de Araújo RT, Teixeira MA. O cuidado de enfermagem ao RN pré-termo em unidade neonatal: perspectiva de profissionais de enfermagem. Rev. Eletr. Enf. [Internet] 2012; 14(3) [acesso em 05 jan 2015]. Disponível: http://www.fen.ufg.br/fen_revista/v14/n3/pdf/v14n3a21.pdf.

12. Bittencourt RM, Gaiva MA, Rosa MKO. Perfil dos recursos humanos das unidades de terapia intensiva neonatal de Cuiabá, MT. Rev. Eletr. Enf. [Internet] 2010; 12(2) [acesso em 06 jan 2015]. Disponível: https://www.fen.ufg.br/ fen_revista/v12/n2/v12n2a05.htm

13. Vieira GB, Cavalcante DS, Ponce de Leon CGRM, Ribeiro LM, Fonseca LMM, Menezes KM. Primeiros passos da educação permanente em Enfermagem de uma unidade de terapia intensiva neonatal. Rev enferm UFPE on line. [Internet] 2014; 8(4) [acesso em 12 jan 2015]. Disponível: http://www.revista.ufpe.br/revistaenfermagem/ index.php/revista/article/download/4162/8836.

14. Caetano EA, Lemos NRF, Cordeiro SM, Pereira FMV, Moreira DS, Buchhorn SMM. The new-born with pain: the role of the nursing team. Esc. Anna Nery. [Internet] 2013; 17(3) [acesso em 12 jan 2015]. Disponível: http:// dx.doi.org/10.1590/S1414-81452013000300006.

15. Costa P, de Camargo PP, Bueno M, Kimura AF. Measuring pain in neonates during placement of central line catheter via peripheral insertion. Acta paul. enferm. [Internet] 2010; 23(1) [acesso em 12 jan 2015]. Disponível: http://dx.doi.org/10.1590/S0103-21002010000100006. 
16. Santos LM, Pereira MP, dos Santos LFN, de Santana RCB. Avaliação da dor no recém-nascido prematuro em Unidade de terapia Intensiva. Rev. bras. enferm. [Internet] 2012; 65(1) [acesso em 12 jan 2015]. Disponível: http:// dx.doi.org/10.1590/S0034-71672012000100004.

17. Amaral JB, Resende TA, Contim D, Barichello E. The nursing staff in the face of pain among preterm newborns. Esc. Anna Nery. [Internet] 2014; 18(2). [acesso em 12 jan 2015]. Disponível: http://dx.doi.org/10.5935/14148145.20140035.

18. Mendes LC, Fontenele FC, Dodt RCM, Almeida LS, Cardoso MVLML, da Silva CBG. A dor no recém-nascido na unidade de terapia intensiva neonatal. Rev enferm UFPE on line. [Internet] 2013; 7(11) [acesso em 1 mar 2015]. Disponível: http://www.revista.ufpe.br/revistaenfermagem/index.php/revista/article/download/3007/7735.

19. Gaiva MAM, Silva FB, Azevedo FM, Rubira EA. Procedimentos dolorosos em recém-nascidos prematuros em unidade terapia intensiva neonatal. Arq. Ciênc. Saúde. [Internet] 2014; 21(1) [acesso em 08 dez 2014]. Disponível: http://repositorio-racs.famerp.br/racs_ol/vol-21-1/ID-576-21(1)-(Jan-Mar-2014).pdf.

20. Lélis ALPA, Farias LM, Cipriano MAB, Cardoso MVLML, Galvão MTG, Caetano JA. Cuidado humanístico e percepções de enfermagem diante da dor do recém-nascido. Esc. Anna Nery. [Internet] 2011; 15(4) [acesso em 1 mar 2015]. Disponível: http://dx.doi.org/10.1590/S1414-81452011000400006.

21. da Silva RC, Ferreira MA. Características dos enfermeiros de uma unidade tecnológica: implicações para o cuidado de enfermagem. Rev. bras. enferm. [Internet] 2011; 64(1) [acesso em 1 mar 2015]. Disponível: http:// dx.doi.org/10.1590/S0034-71672011000100015. 